\title{
Repeat Measurements and Metrics for Nonlinear Model Development
}

\author{
Kate A. Remley, Member, IEEE ${ }^{1}$, Jeffrey A. Jargon, Senior Member, IEEE ${ }^{1}$, Dominique Schreurs,

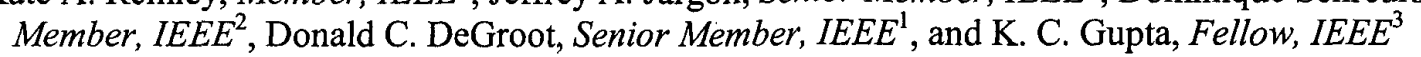

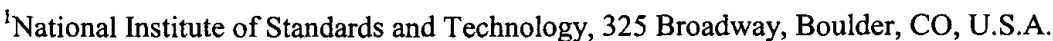 \\ ${ }^{2}$ K.U.Leuven, Div. ESAT-TELEMIC, Kasteelpark Arenberg 10, B-3001 Leuven, Belgium \\ ${ }^{3}$ Dept. of Electric and Electronics Engineering, University of Colorado, Boulder, CO, U.S.A.
}

\begin{abstract}
We develop a method to study nonlinear models using metrics in conjunction with repeat measurements. We illustrate this procedure by investigating the performance of three types of measurement-based nonlinear circuit models using two different metrics.
\end{abstract}

\section{INTRODUCTION}

We generally evaluate the ability of an electrical circuit model to predict circuit behavior with metrics, also called error functions or optimizing functions [1-3]. For example, engineers will compute the difference between a modelis prediction and a set of reference data (often a measurement) and apply a metric such as a sum of squares to this difference. The single scalar quantity generated by the metric is intended to summarize model performance.

In the present work, we extend the use of metrics to evaluate deterministic models that are used to predict measurements that have an inherent stochastic component. Rather than looking at single values of a metric, we look at both the mean and the distribution obtained by applying the metric to a set of repeated measurements. We will demonstrate that this additional information gives insight into model performance, aiding in the development of robust measurement-based models that can predict measurements under realistic operating conditions.

While this technique may be applied to the evaluation of both linear and nonlinear circuit models, the extra information arising from the distribution of measured values and their corresponding metrics is particularly helpful in the evaluation of nonlinear circuit models. For nonlinear circuits, the state of the measurement system itself will affect the circuit response, as discussed in [4]. For example, even slight changes in a measurement systemis output impedance will cause a corresponding change in the excitation across the nonlinear device, changing the circuitis operating point. Thus, a measurement-based nonlinear circuit model must characterize device behavior accurately, not at just a single point, but over a realistic range of operating conditions around the nominal operating point.

U.S. Government work not protected by U.S. copyright
In the following, we describe the procedure we use to calculate the metrics and provide model evaluation examples for three types of deterministic, measurementbased models. Finally, we demonstrate the use of our metrics in a measurement round-robin application. In this example, we see that the metrics alert us to measured data with significant drift.

\section{PROCEDURE}

We first perform many measurements of an electrical circuit under a single operating condition. This set of measurements forms our reference data set. For each measurement in the data set, we generate a model prediction and compute the value of a metric that quantifies the difference between the reference data and the modelís prediction. We complete this procedure for all of the measurements in the reference data set and plot a histogram of the metric values. The mean value and shape of the histogram give us additional information to better quantify candidate prediction models.

In the present work, we use a set of reference data acquired from repeated nonlinear vector network analyzer (NVNA) [5-7] measurements made on a waferlevel diode circuit. We generate predictions of the measured data using three types of models: SPICE-based compact models, and time- and frequency-domain behavioral models [8-10]. From these predictions we calculate values of two types of metrics for each data point and plot them in histogram form.

To illustrate the use of these metrics in model evaluation, we first design an optimized model within each model type and then intentionally degrade the model. This provides a range of agreement with measured results that we are able to detect with the metrics. We next describe the measured reference data, the models, and the metrics used in this study.

\section{A. Reference Data}

We use a set of 100 repeated measurements collected on an NVNA over eight hours. RF input power is +3 $\mathrm{dBm}$ on wafer, the fundamental frequency is $900 \mathrm{MHz}$, and we collect data up to $20 \mathrm{GHz}$. 


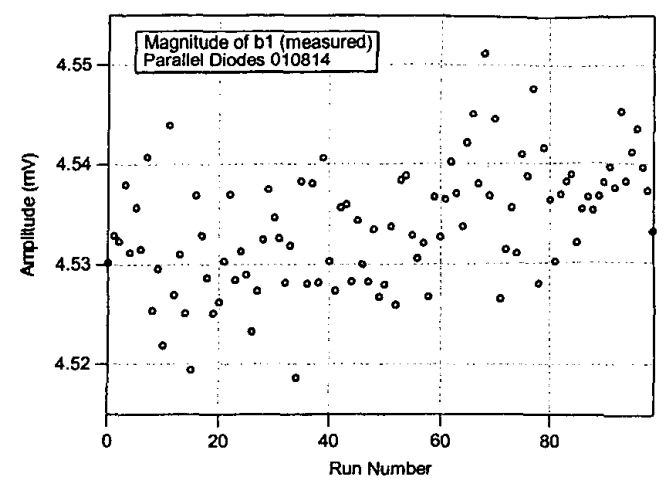

Fig. 1. The magnitude of the measured scattered travelling voltage wave at port $1,\left|b_{1}\right|$, at the fundamental frequency, $f_{0}$.

The wafer-level nonlinear circuit we use in these measurements consists of two parallel Schottky diodes connected to probe pads by $0.5 \mathrm{~mm}$ coplanar waveguide (CPW) lines. The two-port diode circuit is fabricated on an alumina substrate by bonding the beam-lead diode package to a gold metal layer. The diode is forward biased by $0.2 \mathrm{~V}$. Figure 1 shows the magnitude of the scattered travelling voltage measured wave at port $1\left(b_{1}\right)$ for the reference data set at the fundamental frequency. For purposes of demonstration, we assume here that variation in $b$ is due to variation in the incident wave variable $a$ only and is not due to measurement error. In reality the distribution of the $b$ data is due to both of these effects.

\section{A. Models}

Recall that we use three types of models. For each model type, we produce an "optimized" model and then degrade the model in two subsequent designs to see how its prediction of the measured data is affected. The inputs to the model consist of the measured port 1 and port 2 input wave variables $a_{1}$ and $a_{2}$ [4] at DC, the fundamental, and harmonics up to $20 \mathrm{GHz}$.

1. Compact Models: Three SPICE-based models described below are based on the diode manufacturerís parameters. The model Compact Opt is optimized in the time domain by eye to the first measurement in our reference data set. Model Compact $R s C j$ is the same as model Compact Opt with an increase in both $C_{\mathrm{j} 0}$ and $R_{\mathrm{s}}$. Model Compact Phase is the same as model Compact $R s C j$ with an increased CPW interconnect length that introduces an additional phase shift error.

2. Frequency-Domain ANN Models (FD-ANN): Artificial Neural Network (ANN) models $[9,10]$ map input to output variables using training (design) data and a set of weighting functions. ANNs act well as interpolators, and thus require training data that covers the range of expected measurements. We train threelayer ANNs with NVNA data measured at power levels above and below our reference data set level of $+3 \mathrm{dBm}$. The inputs to the trained model consist of the measured input wave variables $a_{1}$ and $a_{2}$ through the first four harmonics. The optimized model, $F D-A N N$ Opt, uses four power levels for training: two above and two below the reference level $(2.90,2.94,3.06$, and $3.10 \mathrm{dBm})$. This model design has five hidden neurons (the weighting functions are applied at the input and output of the hidden neuron layers). Model $F D-A N N$ 4-1 uses the same four power levels, but only one hidden neuron. Model $F D-A N N$ 2.5 uses two power levels (2.9 and 3.1 $\mathrm{dBm}$ ) for training, and has five hidden neurons.

3. Time-Domain Behavioral Models Using ANNs (SVAR): These behavioral models [8] are developed by finding state variable equations of a nonlinear device. Large-signal measurements provide samples of the state variables over the expected range of operating conditions. State variable equations are then generated by fitting these sample points with ANNs. In this study, these models are developed using the same two power levels as $F D-A N N 2-5(2.9$ and $3.1 \mathrm{dBm})$. Model $S V A R$ $O p t$ uses 6 hidden neurons for the ANN, model SVAR 23 uses three hidden neurons, and model SVAR Phase introduces a phase shift error similar to model Compact Phase.

\section{B. Metrics}

While there are many types of metrics used in electrical circuit evaluation [11], we select two:

- The Natural Metric:

$S_{N}\left(b, b^{p}\right)=\left|b_{0}-b^{p} 0\right|^{2}+2 \sum_{i=1}^{N}\left|b_{i}-b^{p}\right|^{2}$

- A Weighted Metric:

$$
S_{W}\left(b, b^{p}\right)=\sum_{i=0}^{N} \frac{\left|b_{i}\right|}{\sum_{j=0}^{N}\left|b_{j}\right|} \mid b_{i}-b^{p_{i} \mid}
$$

Here, $b_{i}$ refers to the $i^{\text {th }}$ harmonic of the measured scattered wave variable $b, b^{p_{i}}$ refers to the predicted value, and $N$ represents the number of harmonics. The units of the natural metric are voltage squared, while the units of the weighted metric are volts. The natural metric is so called because of its equivalence in both the time and frequency domains. The weighted metric puts more emphasis on harmonics with more power. 


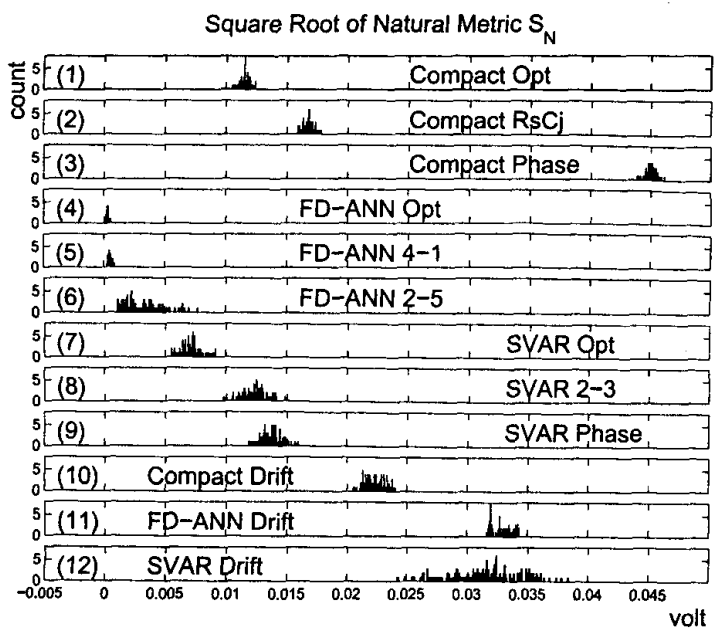

Fig. 2: Histograms of the square root of the natural metric for the nine model designs and the data with drift discussed in Section IV.

\section{MODEL EVALUATION}

Histograms of the metric values obtained with the nine different model types described above are shown in Fig. 2 (square root of natural metric) and Fig. 3 (weighted metric). We take the square root of the natural metric so that the two plots have the same units.

A shift in the mean of a histogram to the right indicates worse agreement between measured and predicted data. We see that the optimized model for each model type has the lowest mean, and that the metrics accurately detect deteriorating model quality for all three model types: compact, FD-ANN, and state variable models. We observe that the introduction of systematic errors in the models results in a large shift of the mean to the right; the use of fewer neurons results in a small shift of the mean to the right; and insufficient training results in an increased spread.

The metrics also give us insight into how various model parameters affect model performance. For example, we see that the phase shift error incorporated into the models Compact Phase (line 3) and SVAR Phase (line 9) has a more detrimental effect on the modelsí ability to predict measured data than other types of model design errors. In the case of the FD-ANNs, we see that using fewer neurons ( $F D-A N N$ 4-1, line 5) has less effect on overall model accuracy than using less training data ( $F D-A N N 2-5$, line 6).

Note that while one model type may appear to more accurately predict these particular sets of measured data,

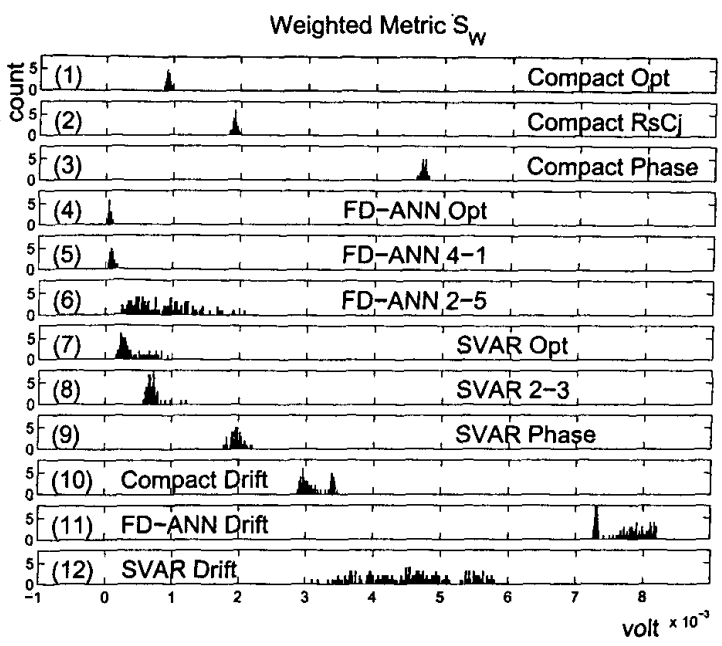

Fig. 3: Histograms of the weighted metric for the nine model designs and the data with drift discussed in Section IV.

another model type may be more robust in terms of predicting measured data at other power/bias levels. Thus, we draw no conclusion from the present work on the overall quality of one model type over another. However, within each model type, we can easily resolve and rank model performance using this method.

\section{APPLICATION TO ROUND-ROBIN SCENARIO}

We tested the two metrics in a measurement round robin scenario similar to one currently under development at NIST [4, 12]. The round robin is intended to quantify differences in NVNA measurements. Here we use the optimized models developed for each model type to predict measurements that contain a significant amount of drift.

As discussed above, our models were designed (trained) using data with input/output relationships similar to those we expect to encounter when the models are used in our application. An example is shown in Fig. 4 , where the black circles correspond to the incident $\left(a_{1}\right)$ and scattered $\left(b_{1}\right)$ waves for the reference data set discussed in Section II. We expect all of the measurements in the round robin to follow a similar input/output relationship. In this example, however, we obtain a measurement where the scattered data contains significant drift caused by changes in the RF path over time (the red triangles in Fig. 4). This particular input/output relationship was not used in the design of our models. Do the metrics detect a difference for this set of data? 


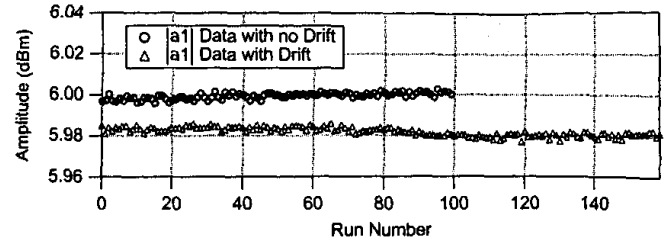

(a)

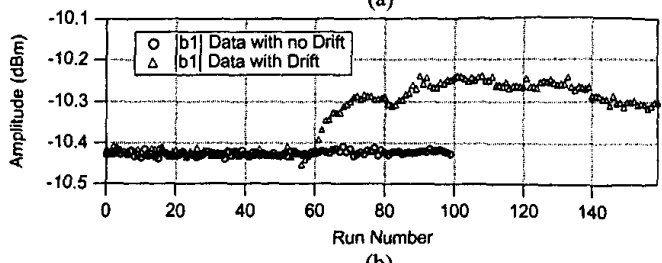

(b)

Fig. 4: Magnitude of the measured incident $(a)$ and scattered (b) waves at the fundamental. The black circles correspond to the reference data set described in Section II. The red triangles correspond to the data with drift used in the metric comparison of Section IV.

Metric values for the data with drift, using our optimized models, are labeled i Driftî in Figs. 2 and 3 (lines 10,11, and 12). We see that both metrics detect that the input/output relationship is different for this set of data compared with their training data, shown as a shift to the right and an increase in the histogram spread.

While both metrics indicate discrepancies between the modeled and measured data, we notice a bimodal distribution in the histograms corresponding to the weighted metric. The left-most peak in the bimodal distribution corresponds to the modelis relatively accurate prediction of the first 60 or so data points (see Fig. 4(b)). The right-most peak corresponds to the modelis prediction of measured data that has drifted outside the range over which the model was trained. Thus, for this particular application, the weighted metric may yield more detailed information regarding the measured data.

\section{SUMMARY}

We have shown how examination of a distribution of metric values can be used to evaluate models. The method can aid in the development of robust measurement-based models that can predict or discern measured behavior under realistic operating conditions. We presented an example showing possible application to an NVNA round robin, and demonstrated that these particular metrics give useful information not available from single values of the metric. We plan to continue work in this area, incorporating temperature effects into the models and investigating other metrics.

\section{REFERENCES}

[1] M. A. Schamberger and A. K. Sarma, "A generalized electromagnetic optimization procedure for the design of complex interacting structures in hybrid and monolithic microwave integrated circuits," IEEE MTT-S Int. Microwave Symp. Dig., pp. 1191-1194, June 1995

[2] C. van Niekerk, P. Meyer, D. Schreurs, and P. B. Winson, "A robust integrated multibias parameterextraction method for MESFET and HEMT models," IEEE Trans. Microwave Theory Tech., vol. 48, pp. 777786, May 2000.

[3] J. W. Bandler, N. Georgieva, M. A. Ismail, J. E. RayasSanchez, and Q.-J. Zhang, "A generalized space-mapping tableau approach to device modeling," IEEE Trans. Microwave Theory Tech., vol. 49, pp. 67-79, Jan. 2001.

[4] K. A. Remley, D. C. DeGroot, J. A. Jargon, and K. C. Gupta, "A method to compare vector nonlinear network analyzers," IEEE MTT-S Int. Microwave Symp. Dig., pp. 1667-1670, May 2001.

[5] G. Kompa and F. van Raay, "Error-corrected large-signal waveform measurement system combining network analyzer and sampling oscilloscope capabilities," IEEE Trans. Microwave Theory Tech., vol. 38, pp. 358-365, Apr. 1990

[6] M. Demmler, P. J. Tasker, and M. Schlechtweg, "Onwafer large signal power, S-parameter and waveform measurement system," Proc. 3rd Int. Workshop on Integrated Nonlinear Microwave and Millimeterwave Circuits, pp. 153-158, 1994.

[7] J. Verspecht, P. Debie, A. Barel, and L. Martens, "Accurate on wafer measurement of phase and amplitude of the spectral components of incident and scattered voltage waves at the signal ports of a nonlinear microwave device," IEEE MTT-S Int. Microwave Symp. Dig., pp. 1029-1032, 1995.

[8] D. Schreurs, J. Wood, N. Tufillaro, D. Usikov, L. Barfor, and D. E. Root, "The construction and evaluation of behavioral models for microwave devices based on timedomain large-signal measurements," Proc. IEEE International Electron Devices Meeting (IEDM), pp. 819 . $822,2000$.

[9] K. C. Gupta, "EM-ANN models for microwave and millimeter-wave components," presented at "Applications of ANNs to Microwave Design" Workshop, IEEE MTT-S Int. Microwave Symp., June 1997.

[10] J. A. Jargon, K. C. Gupta, and D. C. DeGroot, "Artificial neural network modeling for improved on-wafer OSLT calibration standards," Int. J. RF and Microwave CAE, vol. 10, pp. 319-328, 2000.

[11] C. F. Gerald and P. O. Wheatley, Applied Numerical Analysis. Reading, MA: Addison-Wesley, 1992.

[12] D. C. DeGroot, J. A. Jargon, K. A. Remley, and K. C. Gupta, "Verifcation technique for large-signal, nonlinear network analysis," presented at "Going Beyond SParameters" Workshop, European Microwave Conference, London, 2001. 